Published in final edited form as:

Brain Stimul. 2017 ; 10(1): 51-58. doi:10.1016/j.brs.2016.10.001.

\title{
Direct current stimulation modulates LTP and LTD: activity dependence and dendritic effects
}

\author{
Greg Kronberg ${ }^{1}$, Morgan Bridi ${ }^{2,3}$, Ted Abel ${ }^{2}$, Marom Bikson ${ }^{1}$, and Lucas C. Parra ${ }^{1}$ \\ ${ }^{1}$ Department of Biomedical Engineering; The City College of New York; New York, NY,10031; \\ USA \\ ${ }^{2}$ Department of Biology; University of Pennsylvania; Philadelphia, PA, 19104;USA
}

\begin{abstract}
Background-Transcranial direct current stimulation (tDCS) has been reported to improve various forms of learning in humans. Stimulation is often applied during training, producing lasting enhancements that are specific to the learned task. These learning effects are thought be mediated by altered synaptic plasticity. However, the effects of DCS during the induction of endogenous synaptic plasticity remain largely unexplored.
\end{abstract}

Objective/Hypothesis-Here we are interested in the effects of DCS applied during synaptic plasticity induction.

Methods-To model endogenous plasticity we induced long-term potentiation (LTP) and depression (LTD) at Schaffer collateral synapses in CA1 of rat hippocampal slices. Anodal and cathodal DCS at $20 \mathrm{~V} / \mathrm{m}$ were applied throughout plasticity induction in both apical and basal dendritic compartments.

Results-When DCS was paired with concurrent plasticity induction, the resulting plasticity was biased towards potentiation, such that LTP was enhanced and LTD was reduced. Remarkably, both anodal and cathodal stimulation can produce this bias, depending on the dendritic location and type of plasticity induction. Cathodal DCS enhanced LTP in apical dendrites while anodal DCS enhanced LTP in basal dendrites. Both anodal and cathodal DCS reduced LTD in apical dendrites. DCS did not affect synapses that were weakly active or when NMDA receptors were blocked.

Conclusions-These results highlight the role of DCS as a modulator, rather than inducer of synaptic plasticity, as well as the dependence of DCS effects on the spatial and temporal properties of endogenous synaptic activity. The relevance of the present results to human tDCS should be validated in future studies.

Corresponding Author: Greg Kronberg, The City College of New York Center for Discovery and Innovation, 85 St. Nicholas Terrace, Rm 3121, New York, NY 10031, 212-650-8876, gregkronberg@gmail.com.

${ }^{3}$ Present address: Laboratory of Neural Circuits and Behavior, Hussman Institute for Autism, Baltimore MD 21201

Publisher's Disclaimer: This is a PDF file of an unedited manuscript that has been accepted for publication. As a service to our customers we are providing this early version of the manuscript. The manuscript will undergo copyediting, typesetting, and review of the resulting proof before it is published in its final citable form. Please note that during the production process errors may be discovered which could affect the content, and all legal disclaimers that apply to the journal pertain.

Declaration of interest: CUNY has patents on brain stimulation with Marom Bikson and Lucas Parra as inventors. MB and LP have equity in Soterix Medical Inc. 


\section{Keywords}

tDCS; synaptic plasticity; BCM theory; dendrite; membrane polarization; NMDA receptor; activity dependent

\section{Introduction}

Transcranial direct current stimulation (tDCS) applies a weak constant current of $2 \mathrm{~mA}$ or less across the scalp. This apparently simple technique is currently under investigation for a wide variety of conditions, including psychiatric disorders, neurorehabilitation and cognitive enhancement [1]-[3]. Stimulation is often paired with a training task, leading to task-specific enhancements in learning performance [1], [4]. Despite the observation of pharmacological, neuro-physiological and imaging effects in humans [5] and animals [6], a coherent picture of the relevant cellular mechanisms is yet to emerge.

Learning and memory are thought to be mediated by synaptic plasticity [7] and training paradigms in humans presumably influence learning by inducing plasticity [8]. Despite the common practice of applying tDCS during training, cellular effects of DCS applied during endogenous plasticity induction remain largely unexplored. Instead, the majority of research has analyzed effects when DCS precedes plasticity induction [9]-[11], or is paired with endogenous activity otherwise not known to induce plasticity [12]-[14]. Here we are interested in the effects of DCS applied during training, i.e. concurrent with synaptic plasticity induction. As a model of endogenous synaptic plasticity, we induced long-term potentiation (LTP) and depression (LTD) using canonical protocols (pulse trains delivered to Schaffer collateral synapses in CA1 of rat hippocampal slices). By sweeping across induction frequencies we capture a frequency-response function (FRF), which has been widely used to study the predictions of the Bienenstock, Cooper and Munro (BCM) theory of synaptic plasticity. Here we show that DCS can shift the FRF, facilitating LTP and diminishing LTD, similar to BCM-like metaplasticity [15].

A prevailing mechanistic explanation is that tDCS produces shifts in cortical excitability, with anodal stimulation increasing excitability and cathodal stimulation decreasing excitability [5]. This excitability hypothesis is rooted in physiological evidence that DCS modulates membrane potential at neuronal somas, leading to changes in firing rate and timing [16]-[20]. Based on these observations, anodal and cathodal tDCS are often assumed to produce LTP and LTD-like effects, respectively, for an entire brain region [21]-[24]. However, this reasoning ignores the gradient of membrane polarization induced in any neuron during DCS and the role of endogenous synaptic activity in determining effects.

Here we show that DCS effects vary greatly within a small population of neurons, depending on dendritic location and endogenous synaptic activity. Both anodal and cathodal DCS facilitated LTP, but in different dendritic compartments. Moreover, when paired with LTD, DCS effects were independent of polarity. Both anodal and cathodal DCS reduced LTD in the same dendritic compartment. Finally, we show that DCS did not induce plasticity, but rather acted only as a modulator of endogenous synaptic plasticity. Our results motivate a 
more nuanced approach, which accounts for the properties of endogenous synaptic activity in predicting DCS effects.

\section{Materials and Methods}

All animal experiments were carried out in accordance with guidelines and protocols approved by the Institutional Animal Care and Use Committee (IACUC) at The City College of New York, CUNY (Protocol No: 846.3).

Hippocampal brain slices were prepared from male Wistar rats aged 3-5 weeks old, which were deeply anaesthetized with ketamine $\left(7.4 \mathrm{mg} \mathrm{kg}^{-1}\right)$ and xylazine $\left(0.7 \mathrm{mg} \mathrm{kg}^{-1}\right)$ applied I.P., and killed by cervical dislocation. The brain was quickly removed and immersed in chilled $\left(2-6^{\circ} \mathrm{C}\right)$ artificial cerebrospinal fluid (ACSF) containing (in $\mathrm{mM}$ ): $\mathrm{NaCl}, 125 ; \mathrm{KCl}$, 4.4; $\mathrm{NaH}_{2} \mathrm{PO}_{4}, 1 ; \mathrm{MgSO}_{4}, 1.5 ; \mathrm{CaCl}, 2.5 ; \mathrm{NaHCO}_{3}, 26 ;$ D-glucose, 10 ; bubbled with a mixture of $95 \% \mathrm{O}_{2}-5 \% \mathrm{CO}_{2}$. Transverse slices ( $400 \mu \mathrm{m}$ thick) were cut using a vibrating microtome (Campden Instruments) and transferred to a holding chamber for at least $1 \mathrm{~h}$ at ambient temperature. Slices were then transferred to a fluid-gas interface chamber (Harvard Apparatus) perfused with warmed ACSF $\left(30.0 \pm 0.1^{\circ} \mathrm{C}\right)$ at $1.0 \mathrm{ml} \mathrm{min}^{-1}$. The humidified atmosphere over the slices was saturated with a mixture of $95 \% \mathrm{O}_{2}-5 \% \mathrm{CO}_{2}$. Recordings started 2-3 h after dissection.

Field excitatory postsynaptic potentials (fEPSPs) were evoked using a platinum-iridium bipolar stimulating electrode placed in either stratum radiatum or stratum oriens of CA1. Recording electrodes made from glass micropipettes pulled by a Sutter Instruments P-97 and filled with ACSF (resistance 1-8 M $\Omega$ ), were placed in either stratum radiatum or stratum oriens approximately $250 \mu \mathrm{m}$ from the stimulating electrode in CA1 to record fEPSPs. fEPSPs were quantified by the average initial slope, taken during the first 0.5 milliseconds after the onset of the fEPSP. Stimulus intensity was set to evoke fEPSPs with $40 \%$ of the maximum slope, which was determined at the onset of recording. Stable baseline fEPSPs were recorded every minute for at least 20 minutes before any plasticity induction was applied. fEPSPs were then recorded again every minute for 60 minutes after plasticity induction. Plasticity was induced by evoking a train of 900 fEPSPs at varying frequency, based on previous studies of bidirectional synaptic plasticity [25]. Induction frequencies were chosen to be $0.5,1,5$, and $20 \mathrm{~Hz}$. DCS was applied throughout plasticity induction, lasting $30,15,3$, and 0.75 minutes for $0.5,1,5$, and $20 \mathrm{~Hz}$ induction, respectively.

DCS was applied between two parallel $\mathrm{Ag}-\mathrm{AgCl}$ wires (1 mm diameter, $12 \mathrm{~mm}$ length) placed in the bath on opposite sides of the brain slice separated by $10 \mathrm{~mm}$ with the recording site approximately equidistant from each wire. DCS wires were connected to a currentcontrolled analog stimulus isolator (A-M Systems) that was controlled by PowerLab hardware and LabChart software (AD Instruments). Slices were oriented such that the somato-dendritic axis of CA1 pyramidal neurons was parallel to the electric field between the DCS wires (Figure 1A). Before each recording, DCS current intensity was calibrated to produce a $20 \mathrm{~V} / \mathrm{m}$ electric field across each slice (typically $100-200 \mu \mathrm{A}$ ) by adjusting the current so that two recording electrodes separated by $0.8 \mathrm{~mm}$ in the slice measured a voltage difference of $16 \mathrm{mV}(16 \mathrm{mV} / 0.8 \mathrm{~mm}=20 \mathrm{~V} / \mathrm{m})$. 
For NMDAR antagonist experiments, $100 \mu \mathrm{M}$ MK-801 (Sigma Aldrich) was included in the ACSF perfused in the recording chamber throughout the experiment. Because MK-801 is an open channel blocker, baseline fEPSPs were recorded for at least 40 minutes to ensure complete blockade of NMDAR channels [26].

Data acquisition and stimulation waveforms were controlled with PowerLab hardware and LabChart software (AD Systems). Extracellular fEPSPs were amplified (100x), low pass filtered $(3 \mathrm{kHz})$, and digitized $(10 \mathrm{kHz})$. Synaptic plasticity was quantified for each slice by taking the average of the last ten fEPSP slopes (51-60 minutes after plasticity induction) and normalizing to the average of baseline fEPSP slopes (20-1 minutes before plasticity induction). All data are reported as the mean \pm standard error of the mean (SEM). The statistical difference between groups (critical value $=0.05$ ) was estimated using two-tailed student's t-tests, after checking for normality in each group (Lilliefors test for normality, $p>$ 0.05 in all cases). Conditions were not repeated in slices taken from the same animal. Reported $\mathrm{n}$ values therefore represent the number of slices and animals used in each condition.

Here we name the polarity of stimulation based on the orientation of DCS relative to pyramidal neurons. Following convention in human tDCS, DCS with the anode closer to CA1 apical dendrites is referred to as anodal stimulation. Conversely, DCS with the cathode closer to CA1 apical dendrites is referred to cathodal stimulation. Importantly, apical dendrites are polarized oppositely from basal dendrites and somas, regardless of DCS polarity [16], [27], [28]. So anodal DCS will depolarize somas and basal dendrites, while hyperpolarizing apical dendrites. Conversely, cathodal DCS will hyperpolarize somas and basal dendrites, while depolarizing apical dendrites (Figure 1A).

Acute effects were determined based on the first response (two responses for paired pulse data) during DCS and were normalized to the average of baseline responses. Fiber volley amplitude was taken as the difference between the trough of the fiber volley and the mean of the two surrounding peaks. Paired pulse ratio was taken as the ratio of the second and first fEPSP slopes during $20 \mathrm{~Hz}$ HFS (50 ms inter-pulse interval) in each condition.

\section{Results}

\section{DCS shifts the frequency-response function}

Trains of synaptic activity have conventionally been used to induce synaptic plasticity in hippocampal slices [25], [29]. As a model of endogenous synaptic plasticity, trains of 900 pulses at varying frequencies $(0.5,1,5,20 \mathrm{~Hz})$ were applied to the Schaffer collateral pathway synapsing on CA1 apical dendrites. Low frequency stimulation (LFS) generated LTD (0.5 Hz: 84.1 $\pm 2.7 \%$, p <0.001, n=10; $1 \mathrm{~Hz}: 78.9 \pm 2.9 \%$, p<0.0001, n=9), while high frequency stimulation (HFS) generated LTP (20 Hz: $114.1 \pm 2.7 \%, \mathrm{p}<0.001, \mathrm{n}=13)$, and an intermediate frequency marked the transition between LTD and LTP (5 Hz: 95.9 $\pm 3.7 \%$, $\mathrm{p}=0.30, \mathrm{n}=9$ ). The resulting FRF (Figure 1B) maps the degree of synaptic activity during induction to the degree of resulting synaptic plasticity and is consistent with existing literature [15]. 
DCS was then applied during plasticity induction at each frequency. Our previous experiments with the present preparation demonstrate that cathodal DCS depolarizes CA1 apical dendrites (Figure 1A; Bikson et al. 2004, figure 10), and was therefore expected to facilitate LTP in this dendritic region [29]. DCS significantly attenuated LTD induced by 0.5 $\mathrm{Hz}$ (Figure 1C; 97.5 $\pm 5.9 \%, \mathrm{p}=0.04, \mathrm{n}=8$ ) and $1 \mathrm{~Hz}$ LFS (Figure 1D; 89.2 $\pm 3.1 \%, \mathrm{p}=0.03$, $\mathrm{n}=10$ ) and enhanced LTP induced by $20 \mathrm{~Hz}$ HFS (Figure 1F; $128.8 \pm 4.7 \%, \mathrm{p}=0.01, \mathrm{n}=$ 14). DCS had a smaller effect at $5 \mathrm{~Hz}$ that was not significant (Figure 1E; $102.3 \pm 2.2 \%$, p = $0.14, \mathrm{n}=11$ ), consistent with smaller effects observed previously at the threshold between LTP and LTD [30]-[32]. The resulting DCS FRF was significantly shifted compared to control $(\mathrm{F}=17.93, \mathrm{df}=1, \mathrm{p}<0.0001)$. Similar shifts of the FRF have been associated with enhanced learning in cortex [15], [33].

\section{DCS effects on LTP depend on dendritic location}

DCS is known to modulate the membrane potential of neuronal compartments [16], [19], [27], [28], [34] and dendritic membrane potential is known to be a critical determinant of NMDAR-dependent plasticity [29]. Other DCS effects in humans and animals have been shown to be NMDAR-dependent, and it is widely speculated that tDCS exerts long-term effects through membrane polarization and NMDARs [24]. An important subtlety that is often lost in this discussion is that DCS will simultaneously depolarize and hyperpolarize different compartments within the same neuron. Indeed, previous work from our own group with a similar experimental setup showed that cathodal DCS simultaneously depolarizes CA1 apical dendrites while hyperpolarizing their basal dendrites and soma. Conversely, anodal DCS hyperpolarizes CA1 apical dendrites while depolarizing their basal dendrites and soma [16]. We therefore expected that the effects of anodal and cathodal stimulation would vary with dendritic location. To test this we paired both anodal and cathodal DCS with $20 \mathrm{~Hz}$ HFS in both CA1 apical and basal dendrites. In apical dendrites, cathodal DCS enhanced LTP, while anodal DCS had no significant effect (Figure 2C,D; control: $114.1 \pm 2.7 \%, \mathrm{n}=13$; cathodal: $128.8 \pm 4.7 \%, \mathrm{p}=0.01, \mathrm{n}=14$; anodal: $111.7 \pm 4.5 \%, \mathrm{p}=0.63, \mathrm{n}=8$ ). In basal dendrites, anodal DCS now enhanced LTP while cathodal DCS had no significant effect (Figure 2E,F; control: $148.6 \pm 3.6 \%, \mathrm{n}=10$; cathodal: $142.5 \pm 5.2 \%, \mathrm{p}=0.34, \mathrm{n}=10$; anodal: $180.4 \pm 9.1 \%, \mathrm{p}<0.01, \mathrm{n}=5)$. As expected, the effects of anodal and cathodal DCS were dependent on dendritic location.

\section{DCS effects are polarity dependent for LTP but not LTD}

Anodal and cathodal DCS apply stimulation with opposite polarity and are canonically expected to produce opposite effects [35]. As reported above, we find that cathodal and anodal DCS have asymmetric effects on LTP for a given dendritic location. Moreover, when paired with 1Hz LFS we observe no polarity dependence of effects. LTD is reduced by both anodal and cathodal DCS, i.e. synaptic strength is increased compared to control (Figure 2G,H; control: $78.9 \pm 2.9 \%, \mathrm{n}=9$; cathodal: $89.2 \pm 3.1 \%, \mathrm{p}=0.03, \mathrm{n}=10$; anodal: $95.6 \pm 5.9 \%, \mathrm{n}=8, \mathrm{p}=0.04)$. These results reveal that modulation of synaptic plasticity by DCS depends on both the physical location of concurrently active synapses (basal or apical dendrites) and the rate of their activity (LFS or HFS) (Figure 2B). 


\section{DCS effects require a concurrent endogenous source of NMDAR plasticity}

tDCS is often applied under the assumption that stimulation can induce plasticity where the was none before [5], [6], [12], [23]. However, given the modest effects on membrane potential for typical stimulation intensities [19], [27], [28], we propose that DCS instead acts as a modulator of NMDAR plasticity. DCS would therefore require a concurrent endogenous source of plasticity to modulate. To test this requirement we again applied cathodal DCS, but removed endogenous NMDAR-dependent plasticity in two ways: first by weakening synaptic activity to well below the plasticity threshold, and second by directly blocking NMDAR current during strong synaptic activity. When applied during weak synaptic activity (30 pulses, $1 / 60 \mathrm{~Hz}$ ), cathodal DCS had no effect (Figure 3C; control: $99.3 \pm 1.1 \%$, $\mathrm{n}=9$; cathodal DCS: $100.8 \pm 4.0 \%, \mathrm{n}=7 ; \mathrm{p}=0.68$ ). When paired with strong synaptic activity (20 Hz HFS) but NMDARs were blocked with antagonist MK-801, cathodal DCS also had no effect (Figure 3B, control: $92.0 \pm 1.6 \%, n=10$; cathodal DCS: 94.3 $\pm 2.3 \%, n=9 ; p=0.42$ ). These results suggest that DCS may act as a modulator of endogenous synaptic plasticity, rather than an inducer of de novo synaptic plasticity.

\section{Acute effects of DCS on synaptic transmission}

To determine whether the effects of DCS were already apparent in acute synaptic effects , we examined several measures of baseline synaptic transmission. However, one-way ANOVAs yielded no significant effect of stimulation on fEPSP slope (Figure 4A; $\mathrm{F}=0.23, \mathrm{df}=1, \mathrm{p}=0.63, \mathrm{n}=31$ control apical, $\mathrm{n}=9$ control basal, $\mathrm{n}=30$ cathodal apical, $\mathrm{n}=12$ anodal apical, $\mathrm{n}=10$ cathodal basal, $\mathrm{n}=4$ anodal basal, $\mathrm{n}=11 \mathrm{mk}-801$ apical, $\mathrm{n}=6 \mathrm{mk}-801$ cathodal apical), fiber volley amplitude (Figure $4 \mathrm{~B}, \mathrm{~F}=0.33, \mathrm{df}=1, \mathrm{p}=0.57, \mathrm{n}=27$ control apical, $\mathrm{n}=9$ control basal, $\mathrm{n}=26$ cathodal apical, $\mathrm{n}=12$ anodal apical, $\mathrm{n}=9$ cathodal basal, $\mathrm{n}=4$ anodal basal,n=11 mk-801 apical,n=6 mk-801 cathodal apical), or paired pulse ratio (Figure 4C; $\mathrm{F}=0.11, \mathrm{df}=1, \mathrm{p}=0.74, \mathrm{n}=12$ control apical, $\mathrm{n}=10$ control basal, $\mathrm{n}=14$ cathodal apical, $\mathrm{n}=7$ anodal apical, $\mathrm{n}=9$ cathodal basal, $\mathrm{n}=4$ anodal basal, $\mathrm{n}=9 \mathrm{mk}-801$ apical, $\mathrm{n}=6 \mathrm{mk}-801$ cathodal apical).

\section{Discussion}

\section{LTP, LTD, and learning}

There is now strong evidence for a role of both LTP and LTD-like processes in various types of learning and memory [36]-[41]. At the behavioral level, learning is likely to involve both of these processes, with the precise degree of each depending on the specific behavior. For example, some learned behaviors directly require habituation to a familiar stimulus and are specifically dependent on LTD [42], [43]. Other learned behaviors involve formation of new associations and responses to the environment, which require LTP and are eliminated by LTD [36], [40]. We observed that LTP is facilitated in dendrites that are depolarized by DCS. This cellular DCS effect may contribute to enhanced learning when tDCS is paired with training that induces plasticity, such as motor rehabilitation [41], [44]. Indeed, similar shifts in the FRF have been linked to facilitation of learning on both theoretical and experimental grounds [15]. We also observed a reduction of LTD for both stimulation polarities (Figure 2B). One may therefore expect that these effects would disrupt learning that requires LTD. 


\section{Plasticity dependence may underlie task-specific effects}

When tDCS is paired with training, the observed effects are often specific to the trained task [4], [45]. While electrodes are typically placed over an intended target region, it is unlikely that task specificity is solely the result of spatial selectivity of current flow. Even in the most focal tDCS applications (e.g. HD-tDCS), current flow through the brain is diffuse, reaching large swaths of cortex and subcortical structures [46], [47]. Moreover, within any particular brain region, there are likely to be neurons involved in many disparate memory engrams or behaviors. The common assumption that tDCS induces plastic effects indiscriminately [35], or even at weakly active synapses [12], therefore implies broad effects on any cognitive output in the stimulated brain regions. This is at odds with the observed specificity of effects. Instead, to explain task-specificity, tDCS may act as a selective modulator of endogenous synaptic plasticity. Our results support this hypothesis, as DCS had no effect when synaptic input was too weak (Figure 3C) or when NMDARs were blocked during strong synaptic input (Figure 3B), indicating that synaptic efficacy is modulated by DCS only when NMDAR-dependent plasticity is already present. This provides a basis for effects to be task-specific, as synapses associated with the paired task are more likely to be undergoing plasticity and therefore subject to modulation during tDCS. Moreover, this predicts that tDCS effects should be enhanced when paired with tasks that induce synaptic plasticity. Indeed, there is some evidence for this [4], [48]. The precise role of endogenous synaptic activity in DCS effects remains an important area for future research.

\section{A potential role for dendritic membrane polarization}

Under the conventional excitability hypothesis, 'anodal tDCS' is assumed to produce inward cortical current flow, which depolarizes pyramidal neuron somas and hence increases cortical excitability. 'Cathodal tDCS' is soma-hyperpolarizing and thus should reduce cortical excitability [35]. However, it is becoming increasingly clear that this reasoning is an oversimplification, particularly when it comes to long-term effects and learning [49]-[51]. While effects on somatic membrane potential must still be considered, our results here point to a potential role for dendritic membrane polarization in determining DCS effects on synaptic plasticity.

Membrane polarization due to DCS can in principle affect the function of all voltagedependent channels distributed throughout a neuron, particularly the relief of NMDARs from magnesium blockade. This influence may be most pronounced in dendrites, where DCS has been shown to modulate excitability involving multiple voltage-dependent channels [27]. While we do not directly measure membrane polarization in the present experiments, our group has done this previously with the same preparation, showing membrane polarization to be maximal in dendrites (Bikson et al. 2004, figure 10), with opposite polarization in apical and basal dendrites. Indeed we observe modulation of synaptic plasticity that is consistent with this variable dendritic, rather than a singular somatic polarization effect (Figure 2).

Given that DCS effects can vary with dendritic location, tasks that activate synaptic pathways with different dendritic locations may respond differently to the same stimulation. A lack of control over the location of active pathways could therefore lead to highly variable 
results in clinical studies. Indeed variable plasticity in response to tDCS has been linked to differential recruitment of interneurons and dendritic compartments during stimulation [52][54]. Attention to dendritic polarization may therefore help to explain mixed effects observed in tDCS outcomes [49], [50], [55], [56]. Moreover, functional differences between dendritic compartments could be leveraged to optimize stimulation outcomes. For example, "top-down" inputs to sensory cortices are known to arrive predominantly onto pyramidal neuron apical dendrites [57]. Stimulation could therefore be designed to depolarize cortical apical dendrites and bias plasticity at these inputs. Similarly, CA1 basal dendrites receive inputs almost exclusively from $\mathrm{CA} 2 / \mathrm{CA} 3$, while $\mathrm{CA} 1$ apical dendrites also receive input from entorhinal cortex [58]. Targeted depolarization of apical synapses with DCS may therefore selectively enhance entorhinal inputs carrying spatial information [59].

While our results are consistent with a role for DCS-induced dendritic polarization, we cannot rule out differences between basal and apical synapses other than polarization, or the involvement of other cellular DCS effects, such as on inhibitory interneurons, glia, neuromodulators systems, or immune response [6]. Further investigation into the involvement of these systems is an important area for future work. The lack of effects observed on fiber volleys and paired pulse ratio suggest that DCS does not affect recruitment or vesicle release probability at presynaptic terminals. This is expected, as Schaffer collateral fibers are oriented perpendicular to the applied DCS electric field vector. However, the sample size here was too small to conclusively rule out these effects. Similarly, the lack of an acute effect on fEPSP slope may result from smaller sample size [27], weaker fields [16], [53], or a differing preparation [27], compared to previous studies.

\section{Low frequency stimulation effects}

The horizontal axis of the FRF is often equated with the degree of postsynaptic calcium influx during induction. HFS leads to strong calcium influx and triggers LTP, while LFS leads to moderate calcium influx and LTD. Based on this calcium control hypothesis, we expected DCS-induced dendritic polarization to modulate calcium influx through NMDARs and produce horizontal shifts in the FRF [15]. The effects we observe with $1 \mathrm{~Hz}$ LFS may therefore be expected, as a horizontal shift of the FRF in either direction would result in less LTD if $1 \mathrm{~Hz}$ is near the point of maximum LTD (minimum synaptic strength). This interpretation is less adequate in accounting for the effect observed at $0.5 \mathrm{~Hz}$ LFS (Figure 1C), as a left horizontal shift would produce more LTD at $0.5 \mathrm{~Hz}$. However, recent evidence suggests a deviation from the calcium control hypothesis, as LTD can be induced by metabotropic NMDAR function rather than calcium influx [26], [60]-[63]. The calcium and voltage dependence of LTD remains controversial though [62], [64], making it more difficult to interpret results with LFS. We also note that the duration of DCS was particularly long with $0.5 \mathrm{~Hz}$ LFS (30 minutes), potentially producing effects that occur on longer time scales, such as on protein synthesis. For example, priming of BDNF synthesis at the start of DCS [9] may lead to increased BDNF release later on during DCS, which reduces LTD [31]. Future experiments directly measuring calcium influx during these induction protocols may provide some resolution to these issues. 


\section{Effect asymmetry}

Our results demonstrate an asymmetric DCS effect on synaptic plasticity, such that DCS was only able to increase synaptic strength (enhance LTP, reduce LTD). Asymmetries have been found in other animal studies [10], [13] and human studies [1], [50]. In parallel work in our lab, we find an asymmetry in acute DCS effects on cellular excitability. This nonlinearity could be the result of the nonlinear voltage dependence of NMDARs [65] or other voltage dependent channels. Similarly, these asymmetries may reflect floor or ceiling effects of any number of cellular processes, where the endogenous state is such that it can only be modulated in one direction.

\section{Comparison to Human tDCS}

While some human tDCS studies target the hippocampus [66], [67], tDCS is typically thought to influence the cortex [24]. Here, the hippocampal preparation was chosen to facilitate isolation of dendritic compartments, which are clearly segregated in CA1. While there are differences in network wiring and activity, the mechanisms for NMDAR-dependent plasticity appear to be highly conserved between CA1 and cortical pyramidal neurons [29]. We can therefore draw parallels at the level of pyramidal neurons that are undergoing NMDAR-dependent plasticity during stimulation, but our observations should be validated with cortical preparations in the future.

Electric fields produced in the cortex during tDCS are thought to be less than $1 \mathrm{~V} / \mathrm{m}$ [47]. Based on acute effects in previous studies we expected that a $1 \mathrm{~V} / \mathrm{m}$ field may produce $1 \%$ effects on synaptic efficacy [20], [28]. In pilot LTP/LTD experiments, we observed approximately $15 \%$ variability in plasticity between slices. Assuming a linear dose response, which previous animal studies have demonstrated [20], [28], a power analysis (power $=0.8$, $\mathrm{p}=0.05$ ) estimated that $20 \mathrm{~V} / \mathrm{m}$ fields would be required to resolve effects with a reasonable number of animals ( $\mathrm{n}=9$ for $20 \mathrm{~V} / \mathrm{m}, \mathrm{cf} . \mathrm{n}>3000$ for $1 \mathrm{~V} / \mathrm{m}$ fields). Indeed our results are consistent with $\sim 1 \%$ modulation of synaptic plasticity per $1 \mathrm{~V} / \mathrm{m}$ DCS (we observe 10-20\% modulation with $20 \mathrm{~V} / \mathrm{m}$ ). However, the assumption that effects of DCS scale linearly with electric field magnitude still requires validation for synaptic plasticity experiments. The $\sim 1 \%$ modulation that might be expected in humans is likely functionally relevant, as a $1 \%$ modulation of fEPSP plasticity can reflect binary plasticity events at hundreds of synapses per pyramidal neuron [68], [69].

tDCS is typically applied for up to 20 minutes in humans [5]. Here we aimed to study the interaction of DCS with canonical synaptic plasticity induction protocols. Stimulation was therefore only applied during induction, and was shorter when combined with more compressed induction paradigms. For example, DCS was only applied for 45 seconds when combined with $20 \mathrm{~Hz}$ plasticity induction. Some time-dependent DCS effects may therefore be excluded here, and future studies should examine whether these effects could be amplified with longer stimulation duration.

\section{Conclusions and context}

DCS is likely to affect many cellular processes simultaneously [6]. Previous studies in animals [9]-[14], [70] and humans [71]-[73] have implicated various effects related to 
synaptic plasticity (NMDAR, BDNF, adenosine, norepinephrine). However, it remains unknown exactly how the DCS electric field interacts with cellular activity to produce these effects. The brain slice preparation used here allows for precise control over the electric field with respect to neuronal morphology and synaptic activity, facilitating a bottom-up approach. Previous work has taken advantage of this to elucidate acute effects on pyramidal neuron somas and axon terminals [28]. While DCS effects have been demonstrated in multiple neuronal compartments [16], [19], [20], [28], [74], the interaction between these compartments can be nonlinear, dynamic, and antagonistic. Here we provide an example where dendritic, rather than somatic polarization "wins" in modulating frequency-dependent plasticity induction. This highlights the importance of dendritic effects, which have received little attention in the DCS literature. Whether dendritic, somatic, or axonal effects dominate though, is likely a function of ongoing neural activity and the orientation of applied fields, which requires further studies. For example, axonal effects may drive plasticity modulation in response to electric fields that are tangential to the cortical surface [28]. The complexity of inter-compartment interactions during DCS warrants computational models and their experimental validation by testing various endogenous activity states, electric field orientations, and neuronal compartments. Despite this complexity, we highlight that DCS may be most effective when paired with learning paradigms that are known of induce synaptic plasticity.

\title{
Acknowledgments
}

This work is supported by NIH grant R01MH092926

\author{
Abbreviations \\ tDCS transcranial direct current stimulation \\ LTP long-term potentiation \\ LTD long-term depression \\ FRF frequency-response function \\ BCM theory theoretical model proposed by Bienenstock, Cooper, and \\ Munro \\ ACSF artificial cerebrospinal fluid \\ fEPSP field excitatory postsynaptic potential \\ HFS high-frequency stimulation \\ LFS low-frequency stimulation
}

\section{References}

1. Coffman BA, Clark VP, Parasuraman R. Battery powered thought: Enhancement of attention, learning, and memory in healthy adults using transcranial direct current stimulation. NeuroImage. Jan.2014 85:895-908. [PubMed: 23933040] 
2. Gomez Palacio Schjetnan A, Faraji J, Metz GA, Tatsuno M, Luczak A. Transcranial Direct Current Stimulation in Stroke Rehabilitation: A Review of Recent Advancements. Stroke Res. Treat. 2013; 2013:1-14.

3. Kuo M-F, Paulus W, Nitsche MA. Therapeutic effects of non-invasive brain stimulation with direct currents (tDCS) in neuropsychiatric diseases. NeuroImage. Jan; 2014 85(Pt 3):948-960. [PubMed: 23747962]

4. Gill J, Shah-Basak PP, Hamilton R. It's the Thought That Counts: Examining the Task-dependent Effects of Transcranial Direct Current Stimulation on Executive Function. Brain Stimulat. Mar; 2015 8(2):253-259.

5. Brunoni AR, Nitsche MA, Bolognini N, Bikson M, Wagner T, Merabet L, Edwards DJ, ValeroCabre A, Rotenberg A, Pascual-Leone A, Ferrucci R, Priori A, Boggio PS, Fregni F. Clinical research with transcranial direct current stimulation (tDCS): Challenges and future directions. Brain Stimulat. Jul; 2012 5(3):175-195.

6. Pelletier SJ, Cicchetti F. Cellular and Molecular Mechanisms of Action of Transcranial Direct Current Stimulation: Evidence from In Vitro and In Vivo Models. Int. J. Neuropsychopharmacol. Jan; 2015 18(2):pyu047-pyu047.

7. Takeuchi T, Duszkiewicz AJ, Morris RGM. The synaptic plasticity and memory hypothesis: encoding, storage and persistence. Philos. Trans. R. Soc. Lond. B. Biol. Sci. Jan.2014 369(1633): 20130288. [PubMed: 24298167]

8. Draganski B, May A. Training-induced structural changes in the adult human brain. Behav. Brain Res. Sep; 2008 192(1):137-142. [PubMed: 18378330]

9. Podda MV, Cocco S, Mastrodonato A, Fusco S, Leone L, Barbati SA, Colussi C, Ripoli C, Grassi C. Anodal transcranial direct current stimulation boosts synaptic plasticity and memory in mice via epigenetic regulation of Bdnf expression. Sci. Rep. Feb.2016 6:22180. [PubMed: 26908001]

10. Ranieri F, Podda MV, Riccardi E, Frisullo G, Dileone M, Profice P, Pilato F, Di Lazzaro V, Grassi C. Modulation of LTP at rat hippocampal CA3-CA1 synapses by direct current stimulation. J. Neurophysiol. Apr; 2012 107(7):1868-1880. [PubMed: 22236710]

11. Rohan JG, Carhuatanta KA, McInturf SM, Miklasevich MK, Jankord R. Modulating Hippocampal Plasticity with In Vivo Brain Stimulation. J. Neurosci. Sep; 2015 35(37):12824-12832. [PubMed: 26377469]

12. Fritsch B, Reis J, Martinowich K, Schambra HM, Ji Y, Cohen LG, Lu B. Direct current stimulation promotes BDNF-dependent synaptic plasticity: potential implications for motor learning. Neuron. Apr; 2010 66(2):198-204. [PubMed: 20434997]

13. Marquez-Ruiz J, Leal-Campanario R, Sanchez-Campusano R, Molaee-Ardekani B, Wendling F, Miranda PC, Ruffini G, Gruart A, Delgado-Garcia JM. Transcranial direct-current stimulation modulates synaptic mechanisms involved in associative learning in behaving rabbits. Proc. Natl. Acad. Sci. Apr; 2012 109(17):6710-6715. [PubMed: 22493252]

14. Monai H, Ohkura M, Tanaka M, Oe Y, Konno A, Hirai H, Mikoshiba K, Itohara S, Nakai J, Iwai Y, Hirase H. Calcium imaging reveals glial involvement in transcranial direct current stimulationinduced plasticity in mouse brain. Nat. Commun. Mar.2016 7:11100. [PubMed: 27000523]

15. Cooper LN, Bear MF. The BCM theory of synapse modification at 30: interaction of theory with experiment. Nat. Rev. Neurosci. Oct; 2012 13(11):798-810. [PubMed: 23080416]

16. Bikson M, Inoue M, Akiyama H, Deans JK, Fox JE, Miyakawa H, Jefferys JGR. Effects of uniform extracellular DC electric fields on excitability in rat hippocampal slices in vitro. J. Physiol. May; 2004 557(Pt 1):175-190. [PubMed: 14978199]

17. Purpura DP, Mcmurtry JG. Intracellular activities and evoked potential changes during polarization of motor cortex. J. Neurophysiol. Jan.1965 28:166-185. [PubMed: 14244793]

18. Radman T, Su Y, An JH, Parra LC, Bikson M. Spike timing amplifies the effect of electric fields on neurons: implications for endogenous field effects. J. Neurosci. Off. J. Soc. Neurosci. Mar; 2007 27(11):3030-3036.

19. Radman T, Ramos RL, Brumberg JC, Bikson M. Role of cortical cell type and morphology in subthreshold and suprathreshold uniform electric field stimulation in vitro. Brain Stimulat. Oct; 2009 2(4):215-228. 228, e1-3. 
20. Reato D, Rahman A, Bikson M, Parra LC. Low-intensity electrical stimulation affects network dynamics by modulating population rate and spike timing. J. Neurosci. Off. J. Soc. Neurosci. Nov; 2010 30(45):15067-15079.

21. Dayan E, Censor N, Buch ER, Sandrini M, Cohen LG. Noninvasive brain stimulation: from physiology to network dynamics and back. Nat. Neurosci. Jun; 2013 16(7):838-844. [PubMed: 23799477]

22. Monte-Silva K, Kuo M-F, Hessenthaler S, Fresnoza S, Liebetanz D, Paulus W, Nitsche MA. Induction of Late LTP-Like Plasticity in the Human Motor Cortex by Repeated Non-Invasive Brain Stimulation. Brain Stimulat. May; 2013 6(3):424-432.

23. Ridding MC, Ziemann U. Determinants of the induction of cortical plasticity by non-invasive brain stimulation in healthy subjects: Induction of cortical plasticity by non-invasive brain stimulation. J. Physiol. Jul; 2010 588(13):2291-2304. [PubMed: 20478978]

24. Stagg CJ, Nitsche MA. Physiological basis of transcranial direct current stimulation. Neurosci. Rev. J. Bringing Neurobiol. Neurol. Psychiatry. Feb; 2011 17(1):37-53.

25. Mayford M, Wang J, Kandel ER, O'Dell TJ. CaMKII regulates the frequency-response function of hippocampal synapses for the production of both LTD and LTP. Cell. Jun; 1995 81(6):891-904. [PubMed: 7781066]

26. Nabavi S, Kessels HW, Alfonso S, Aow J, Fox R, Malinow R. Metabotropic NMDA receptor function is required for NMDA receptor-dependent long-term depression. Proc. Natl. Acad. Sci. U. S. A. Mar; 2013 110(10):4027-4032. [PubMed: 23431133]

27. Andreasen M, Nedergaard S. Dendritic electrogenesis in rat hippocampal CA1 pyramidal neurons: functional aspects of $\mathrm{Na}+$ and $\mathrm{Ca} 2+$ currents in apical dendrites. Hippocampus. 1996; 6(1):79-95. [PubMed: 8878746]

28. Rahman A, Reato D, Arlotti M, Gasca F, Datta A, Parra LC, Bikson M. Cellular effects of acute direct current stimulation: somatic and synaptic terminal effects. J. Physiol. May; 2013 591(Pt 10): 2563-2578. [PubMed: 23478132]

29. Malenka RC, Bear MF. LTP and LTD. Neuron. Sep; 2004 44(1):5-21. [PubMed: 15450156]

30. Hulme SR, Jones OD, Ireland DR, Abraham WC. Calcium-Dependent But Action PotentialIndependent BCM-Like Metaplasticity in the Hippocampus. J. Neurosci. May; 2012 32(20):67856794. [PubMed: 22593048]

31. Ikegaya Y, Ishizaka Y, Matsuki N. BDNF attenuates hippocampal LTD via activation of phospholipase $\mathrm{C}$ : implications for a vertical shift in the frequency-response curve of synaptic plasticity. Eur. J. Neurosci. Jul; 2002 16(1):145-148. [PubMed: 12153539]

32. Lisman JE. Three $\mathrm{Ca}^{2+}$ levels affect plasticity differently: the LTP zone, the LTD zone and no man's land. J. Physiol. Apr; 2001 532(2):285-285. [PubMed: 11306649]

33. Hulme SR, Jones OD, Abraham WC. Emerging roles of metaplasticity in behaviour and disease. Trends Neurosci. Jun; 2013 36(6):353-362. [PubMed: 23602195]

34. Chan CY, Hounsgaard J, Nicholson C. Effects of electric fields on transmembrane potential and excitability of turtle cerebellar Purkinje cells in vitro. J. Physiol. Aug.1988 402:751-771. [PubMed: 3236254]

35. Nitsche MA, Cohen LG, Wassermann EM, Priori A, Lang N, Antal A, Paulus W, Hummel F, Boggio PS, Fregni F, Pascual-Leone A. Transcranial direct current stimulation: State of the art 2008. Brain Stimulat. Jul; 2008 1(3):206-223.

36. Broussard JI, Yang K, Levine AT, Tsetsenis T, Jenson D, Cao F, Garcia I, Arenkiel BR, Zhou F-M, De Biasi M, Dani JA. Dopamine Regulates Aversive Contextual Learning and Associated In Vivo Synaptic Plasticity in the Hippocampus. Cell Rep. Mar; 2016 14(8):1930-1939. [PubMed: 26904943]

37. Connor SA, Wang YT. A Place at the Table: LTD as a Mediator of Memory Genesis. The Neuroscientist. May.2015

38. Dong Z, Bai Y, Wu X, Li H, Gong B, Howland JG, Huang Y, He W, Li T, Wang YT. Hippocampal long-term depression mediates spatial reversal learning in the Morris water maze. Neuropharmacology. Jan.2013 64:65-73. [PubMed: 22732443] 
39. Kuhlman SJ, O'Connor DH, Fox K, Svoboda K. Structural Plasticity within the Barrel Cortex during Initial Phases of Whisker-Dependent Learning. J. Neurosci. Apr; 2014 34(17):6078-6083. [PubMed: 24760867]

40. Nabavi S, Fox R, Proulx CD, Lin JY, Tsien RY, Malinow R. Engineering a memory with LTD and LTP. Nature. Jun; 2014 511(7509):348-352. [PubMed: 24896183]

41. Rioult-Pedotti M-S. Learning-Induced LTP in Neocortex. Science. Oct; 2000 290(5491):533-536. [PubMed: 11039938]

42. Griffiths S, Scott H, Glover C, Bienemann A, Ghorbel MT, Uney J, Brown MW, Warburton EC, Bashir ZI. Expression of Long-Term Depression Underlies Visual Recognition Memory. Neuron. Apr; 2008 58(2):186-194. [PubMed: 18439404]

43. Massey PV, Bashir ZI. Long-term depression: multiple forms and implications for brain function. Trends Neurosci. Apr; 2007 30(4):176-184. [PubMed: 17335914]

44. Kang N, Summers JJ, Cauraugh JH. Transcranial direct current stimulation facilitates motor learning post-stroke: a systematic review and meta-analysis. J. Neurol. Neurosurg. Psychiatry. Apr; 2016 87(4):345-355. [PubMed: 26319437]

45. Bikson M, name A, Rahman A. Origins of specificity during tDCS: anatomical, activity-selective, and input-bias mechanisms. Front. Hum. Neurosci. 2013; 7

46. Borckardt JJ, Bikson M, Frohman H, Reeves ST, Datta A, Bansal V, Madan A, Barth K, George MS. A Pilot Study of the Tolerability and Effects of High-Definition Transcranial Direct Current Stimulation (HD-tDCS) on Pain Perception. J. Pain. Feb; 2012 13(2):112-120. [PubMed: 22104190]

47. Datta A, Bansal V, Diaz J, Patel J, Reato D, Bikson M. Gyri-precise head model of transcranial direct current stimulation: improved spatial focality using a ring electrode versus conventional rectangular pad. Brain Stimulat. Oct; 2009 2(4):201-207. 207, e1.

48. Martin DM, Liu R, Alonzo A, Green M, Loo CK. Use of transcranial direct current stimulation (tDCS) to enhance cognitive training: effect of timing of stimulation. Exp. Brain Res. Oct; 2014 232(10):3345-3351. [PubMed: 24992897]

49. Antal A, Terney D, Poreisz C, Paulus W. Towards unravelling task-related modulations of neuroplastic changes induced in the human motor cortex: Effect of tDCS is modified by mental activity and exercise. Eur. J. Neurosci. Oct; 2007 26(9):2687-2691. [PubMed: 17970738]

50. Jacobson L, Koslowsky M, Lavidor M. tDCS polarity effects in motor and cognitive domains: a meta-analytical review. Exp. Brain Res. Jan; 2012 216(1):1-10. [PubMed: 21989847]

51. Vines BW, Nair D, Schlaug G. Modulating activity in the motor cortex affects performance for the two hands differently depending upon which hemisphere is stimulated. Eur. J. Neurosci. Oct; 2008 28(8):1667-1673. [PubMed: 18973584]

52. Wiethoff S, Hamada M, Rothwell JC. Variability in response to transcranial direct current stimulation of the motor cortex. Brain Stimulat. Jun; 2014 7(3):468-475.

53. Hamada M, Galea JM, Di Lazzaro V, Mazzone P, Ziemann U, Rothwell JC. Two Distinct Interneuron Circuits in Human Motor Cortex Are Linked to Different Subsets of Physiological and Behavioral Plasticity. J. Neurosci. Sep; 2014 34(38):12837-12849. [PubMed: 25232119]

54. Sommer M, Norden C, Schmack L, Rothkegel H, Lang N, Paulus W. Opposite Optimal Current Flow Directions for Induction of Neuroplasticity and Excitation Threshold in the Human Motor Cortex. Brain Stimulat. May; 2013 6(3):363-370.

55. Horvath JC, Carter O, Forte JD. Transcranial direct current stimulation: five important issues we aren't discussing (but probably should be). Front. Syst. Neurosci. 2014; 8

56. Saucedo Marquez CM, Zhang X, Swinnen SP, Meesen R, Wenderoth N. Task-Specific Effect of Transcranial Direct Current Stimulation on Motor Learning. Front. Hum. Neurosci. 2013; 7

57. Harris KD, Mrsic-Flogel TD. Cortical connectivity and sensory coding. Nature. Nov; 2013 503(7474):51-58. [PubMed: 24201278]

58. Amaral, D., Lavenex, P. The Hippocampus Book. Oford University Press; 2007. Neuroanatomy of the Rat Hippocampal Formation; p. 71-76.

59. Brun VH, Leutgeb S, Wu H-Q, Schwarcz R, Witter MP, Moser EI, Moser M-B. Impaired Spatial Representation in CA1 after Lesion of Direct Input from Entorhinal Cortex. Neuron. Jan; 2008 57(2):290-302. [PubMed: 18215625] 
60. Aow J, Dore K, Malinow R. Conformational signaling required for synaptic plasticity by the NMDA receptor complex. Proc. Natl. Acad. Sci. Nov.2015:201520029.

61. Dore K, Aow J, Malinow R. Agonist binding to the NMDA receptor drives movement of its cytoplasmic domain without ion flow. Proc. Natl. Acad. Sci. Nov.2015:201520023.

62. Nabavi S, Fox R, Alfonso S, Aow J, Malinow R. GluA1 trafficking and metabotropic NMDA: addressing results from other laboratories inconsistent with ours. Philos. Trans. R. Soc. B Biol. Sci. Dec; 2013 369(1633):20130145-20130145.

63. Stein IS, Gray JA, Zito K. Non-Ionotropic NMDA Receptor Signaling Drives Activity-Induced Dendritic Spine Shrinkage. J. Neurosci. Sep; 2015 35(35):12303-12308. [PubMed: 26338340]

64. Babiec WE, Guglietta R, Jami SA, Morishita W, Malenka RC, O'Dell TJ. Ionotropic NMDA Receptor Signaling Is Required for the Induction of Long-Term Depression in the Mouse Hippocampal CA1 Region. J. Neurosci. Apr; 2014 34(15):5285-5290. [PubMed: 24719106]

65. Jahr CE, Stevens CF. Voltage dependence of NMDA-activated macroscopic conductances predicted by single-channel kinetics. J. Neurosci. Off. J. Soc. Neurosci. Sep; 1990 10(9):3178-3182.

66. Nikolin S, Loo CK, Bai S, Dokos S, Martin DM. Focalised stimulation using high definition transcranial direct current stimulation (HD-tDCS) to investigate declarative verbal learning and memory functioning. NeuroImage. Aug.2015 117:11-19. [PubMed: 25987365]

67. Boggio PS, Khoury LP, Martins DCS, Martins OEMS, de Macedo EC, Fregni F. Temporal cortex direct current stimulation enhances performance on a visual recognition memory task in Alzheimer disease. J. Neurol. Neurosurg. Psychiatry. Dec; 2008 80(4):444-447. [PubMed: 18977813]

68. O'Connor DH, Wittenberg GM, Wang SS-H. Graded bidirectional synaptic plasticity is composed of switch-like unitary events. Proc. Natl. Acad. Sci. Jul; 2005 102(27):9679-9684. [PubMed: 15983385]

69. Petersen CC, Malenka RC, Nicoll RA, Hopfield JJ. All-or-none potentiation at CA3-CA1 synapses. Proc. Natl. Acad. Sci. U. S. A. Apr; 1998 95(8):4732-4737. [PubMed: 9539807]

70. Reato D, Bikson M, Parra LC. Lasting modulation of in vitro oscillatory activity with weak direct current stimulation. J. Neurophysiol. Mar; 2015 113(5):1334-1341. [PubMed: 25505103]

71. Liebetanz D. Pharmacological approach to the mechanisms of transcranial DC-stimulation-induced after-effects of human motor cortex excitability. Brain. Oct; 2002 125(10):2238-2247. [PubMed: 12244081]

72. Nitsche MA, Fricke K, Henschke U, Schlitterlau A, Liebetanz D, Lang N, Henning S, Tergau F, Paulus W. Pharmacological modulation of cortical excitability shifts induced by transcranial direct current stimulation in humans. J. Physiol. Nov; 2003 553(Pt 1):293-301. [PubMed: 12949224]

73. Rroji O, van Kuyck K, Nuttin B, Wenderoth N. Anodal tDCS over the Primary Motor Cortex Facilitates Long-Term Memory Formation Reflecting Use-Dependent Plasticity. PLOS ONE. May. 2015 10(5):e0127270. [PubMed: 25996937] 


\section{Highlights}

- tDCS is thought to alter cognition through cortical excitability shifts, with anodal and cathodal stimulation producing opposite effects. Here we show that DCS facilitates LTP and diminishes LTD in hippocampal slices. These effects can be polarity independent, as both anodal and cathodal DCS can produce similar effects. Outcomes depended on dendritic location and endogenous synaptic activity, motivating a more nuanced approach to tDCS that considers these factors. 

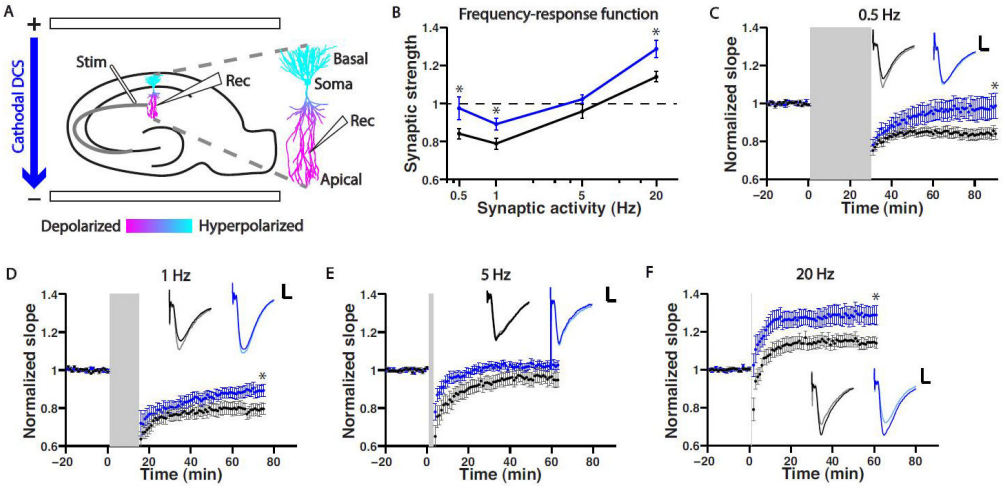

Figure 1. DCS shifts synaptic plasticity in apical dendrites towards potentiation

A: Schematic depicts cathodal DCS of a hippocampal slice, with expected membrane polarization of CA1 pyramidal neuron (enlarged at right; prediction based on computational model as in Rahman et al. 2013). Arrow indicates the direction of positive current flow between electric field wires placed in the recording chamber (horizontal bars above and below hippocampal slice). B: Cathodal DCS (blue) shifts the BCM-like frequency-response function towards potentiation. C-F: DCS applied during plasticity-inducing LFS attenuated LTD (C,D) and enhanced LTP (F), but the effect was not significant near the crossover point between LTD and LTP (E). Sample fEPSP traces are provided for each condition (grey/ black: before/after control; light blue/blue: before/after cathodal; scale bars: $1 \mathrm{mV}, 4 \mathrm{~ms}$ ). Synaptic strength in $(\mathbf{B})$ is the average of the last ten normalized fEPSP slopes in each condition (51-60 minutes post-induction). Grey bars indicate the duration of plasticity induction and concurrent DCS. Data are represented as mean \pm SEM across slices. * $=p<$ 0.05 . 


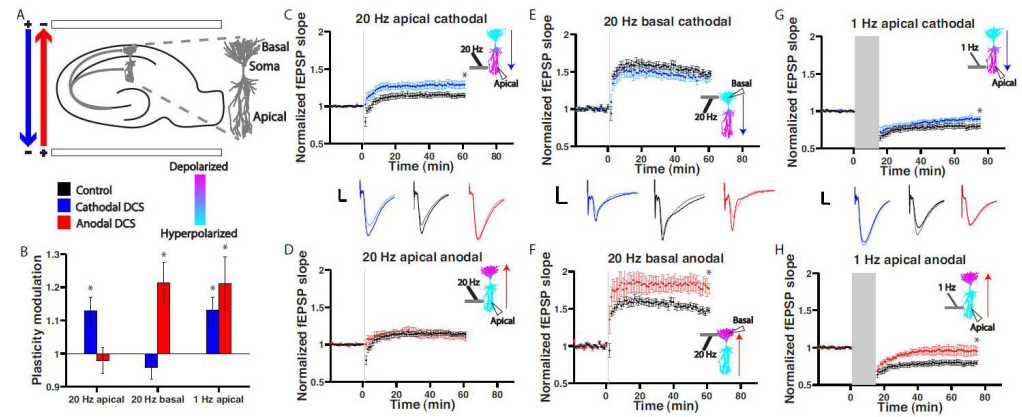

Figure 2. Mixed of effects of anodal and cathodal DCS

A: Schematic depicts anodal (red) and cathodal (blue) DCS of a hippocampal slice. Arrows indicate the direction of positive current flow between DCS electrodes. Reconstruction of a CA1 pyramidal neuron with dendritic compartments labeled at right. B: Modulation of synaptic plasticity depends on polarity, dendritic location and rate of plasticity induction. CD: In apical dendrites cathodal DCS enhances LTP, but anodal has no significant effect. Data in $\mathbf{C}$ is the same as in Figure 1F. E-F: Changing dendritic location to basal dendrites, anodal DCS now enhances LTP, but cathodal DCS has no effect. G-H: Changing induction frequency to $1 \mathrm{~Hz}$ LFS (in apical dendrites), both anodal and cathodal reduce LTD. Data in $\mathbf{G}$ is the same as in Figure 1D. C-H: Example traces for each condition are given in the center of each column (grey/light blue/pink traces are before plasticity induction; black/ blue/red traces are after plasticity induction; scale bars: $1 \mathrm{mV}, 4 \mathrm{~ms}$ ). Insets depict the reconstructed CA1 pyramidal neuron in (A) with expected membrane polarization (prediction based on computational model as in Rahman et al. 2013), induction and recording sites, and orientation of DCS electric field. Grey bars indicate the duration of plasticity induction and concurrent DCS. Plasticity modulation in $(\mathbf{B})$ is the resulting plasticity in each DCS condition normalized to the mean of the plasticity in the corresponding control condition. Data are represented as mean \pm SEM across slices. * $=\mathrm{p}<$ 0.05 . 

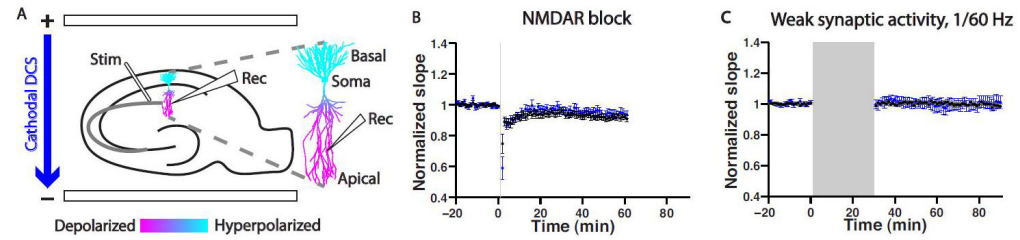

Figure 3. DCS requires an endogenous source of NMDAR plasticity

A: Schematic depicts cathodal DCS (blue) of a hippocampal slice, with expected membrane polarization of CA1 pyramidal neuron (enlarged at right; prediction based on computational model created as described in Rahman et al. 2013). B: Cathodal DCS with $20 \mathrm{~Hz}$ HFS has no effect on synaptic strength when NMDARs are blocked with antagonist MK-801 C: Cathodal DCS applied during synaptic activity that is too weak to induce plasticity (30 pulses at $1 / 60 \mathrm{~Hz}$ ) has no effect on synaptic strength. Grey bars indicate duration of induction and concurrent DCS. Data are represented as mean \pm SEM across slices. 

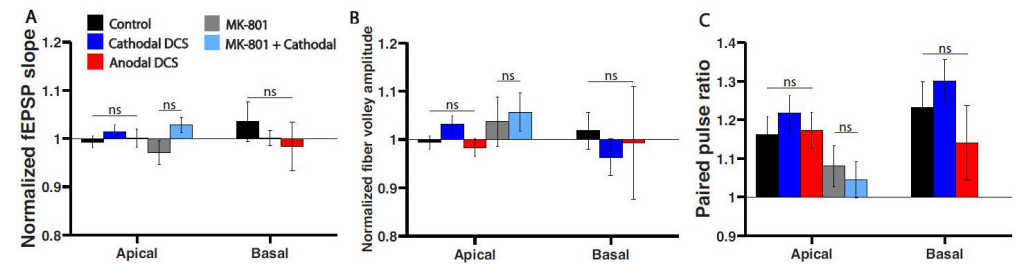

Figure 4. No significant effects on baseline synaptic transmission

DCS had no significant effect on fEPSP slope (A), fiber volley amplitude (B), or paired pulse ratio $(\mathbf{C})$, in apical or basal dendrites, or when NMDARs were blocked with MK-801. Data are represented as mean \pm SEM across slices. $n s=p>0.05$. 